\title{
Lepton masses from a TeV scale in a 3-3-1 model
}

\author{
J. C. Montero, ${ }^{*}$ C. A. de S. Pires, ${ }^{\dagger}$ and V. Pleitez \\ Instituto de Física Teórica, Universidade Estadual Paulista, Rua Pamplona 145, 01405-900 São Paulo, SP Brazil
}

(Received 2 April 2002; revised manuscript received 16 August 2002; published 18 December 2002)

\begin{abstract}
In this work, using the fact that in 3-3-1 models the same leptonic bilinear contributes to the masses of both charged leptons and neutrinos, we develop an effective operator mechanism to generate mass for all leptons. The effective operators have dimension five for the case of charged leptons and dimension seven for neutrinos. By adding extra scalar multiplets and imposing the discrete symmetry $Z_{9} \otimes Z_{2}$ we are able to generate realistic textures for the leptonic mixing matrix. This mechanism requires new physics at the $\mathrm{TeV}$ scale.
\end{abstract}

DOI: 10.1103/PhysRevD.66.113003

PACS number(s): 14.60.Pq, 12.60.-i, 14.60.St

\section{INTRODUCTION}

The smallness of the neutrino masses and the pattern of their mixing, arising from atmospheric and solar neutrino data [1-3], suggest the extension of the standard model. According to those experimental data, the mixing involved in the atmospheric neutrino oscillation is maximal and the mixing involved in the solar neutrino oscillation is large [4,5]. From the theoretical point of view, we dispose already of well established ways where explanations of the smallness of those masses arise naturally. The most popular are the seesaw [6] and the radiative generation [7] mechanisms. Both of them require realistic extensions of the standard model and extra global and/or discrete symmetries. We also can consider effective operators which naturally lead to light neutrinos. This approach has had success in accounting for the neutrino puzzle in the base of the standard model without resorting to drastic fine-tuning.

It was Weinberg [8], and independently Zee and Wilczek [9], who first pointed out in the context of the standard model that the dimension-five effective operator

$$
\frac{1}{\Lambda} \overline{L_{i a}^{c}} L_{j b} \varphi_{k}^{(m)} \varphi_{l}^{(n)}\left(f_{a b m n} \epsilon_{i k} \epsilon_{j l}+f_{a b m n}^{\prime} \epsilon_{i j} \epsilon_{k l}\right)
$$

with $L=\left(\nu_{l}, l\right)_{L}^{T}$, yields naturally light neutrino masses. The success of such an effective operator approach is justified by the expression of the neutrino mass it generates:

$$
M_{a b}^{\nu}=\frac{f_{a b}}{\Lambda}\langle\varphi\rangle^{2},
$$

which is a seesaw relation since $\langle\varphi\rangle \approx 246 \mathrm{GeV}$ and $\Lambda$ is a large characteristic mass. In particular, in this case the realization of such operator turns to be important once it can guide us to all the possible realizations of that mechanism using only the representation content of the standard model with operators of dimension five [10]. Higher dimension op-

\footnotetext{
*Email address: montero@ift.unesp.br

†Email address: cpires@ift.unesp.br

*Email address: vicente@ift.unesp.br
}

erators have already been considered [11], and extensions of the scalar sector of the standard model have also been suggested [12].

In this work we address the problem of generating neutrino and charged lepton masses through effective operators in the context of 3-3-1 models $[13,14]$. In general, in these models, the minimal set of scalar multiplets required to generate the fermion masses consists in three scalar triplets: $\eta=\left(\eta^{0}, \eta_{1}^{-}, \eta_{2}^{+}\right)^{T} \sim(\mathbf{1}, \mathbf{3}, 0), \quad \rho=\left(\rho^{+}, \rho^{0}, \rho^{++}\right)^{T} \sim(\mathbf{1}, \mathbf{3}, 1)$, $\chi=\left(\chi^{-}, \chi^{--}, \chi^{0}\right)^{T} \sim(\mathbf{1}, \mathbf{3},-1)$, and a symmetric sextet $S$ $\sim(\mathbf{1}, 6,0)$. An important feature of this model is that the same leptonic bilinear $\overline{\Psi_{L}^{c}} \Psi_{L}$, where $\Psi_{L}=\left(\nu, l, l^{c}\right)^{T}$, can give a contribution to the masses of both sectors: charged leptons and neutrinos. We explore this feature in order to obtain realistic textures for the mixing matrix in the lepton sector. We work first in a scenario where there are only triplets, like $\eta, \rho$, and $\chi$, and neutral scalar singlets. In this context, depending on the extra symmetry added to the model we obtain realistic texture for the lepton masses. Next we show that a possible realization of this mechanism is one in which heavy scalar sextets and singlet charged leptons $[15,16]$ are added to the model. The scalar sextet which does not gain a vacuum expectation value induces a dimensionfive operator that give mass for the charged leptons; while the dimension-seven operator, which gives mass to the neutrinos, arises from the interactions of neutrinos with the singlet charged leptons and a mixture among the singly charged scalar bosons.

The outline of this work is as follows. In Sec. II we develop a formalism to address the issue of generating the charged lepton and neutrino masses by effective operators in the context of a 3-3-1 model. Next, in Sec. III we use the formalism previously developed to generate realistic scenarios to accommodate lepton masses with appropriate mixing in the lepton sector. In Sec. IV we suggest what are the main ingredients that a more fundamental theory has to have in order to realize such effective operators. We reserve Sec. $\mathrm{V}$ for our conclusions.

\section{THE MECHANISM}

In this section we build effective operators of dimension five for the generation of the charged lepton masses and dimension seven for the case of neutrino masses. In particular, we show that with a dimension-seven operator we attain the 
desired order of the neutrino masses required by the recent experiments, i.e., at the $\mathrm{eV}$ scale. It is interesting to note that the energy scale required to obtain those neutrino masses is of the order of $5 \mathrm{TeV}$.

In the 3-3-1 models of Refs. [13-15] all leptons transform as triplets under the electroweak gauge symmetry:

$$
\Psi_{a L}=\left(\begin{array}{c}
\nu_{a} \\
l_{a} \\
l_{a}^{c}
\end{array}\right)_{L} \sim(\mathbf{1}, \mathbf{3}, 0), \quad a=e, \mu, \tau .
$$

Below we generate the charged lepton masses by using the two scalar triplets $\rho$ and $\chi$, mentioned in the previous section, and the neutrino masses using the triplet $\eta$ and a neutral scalar singlet $\phi$.

In order to generate a realistic texture of the relevant matrices in the lepton sector we will impose appropriate discrete or global symmetries (see the next section).

\section{A. Charged lepton masses}

In the version without the scalar sextet we still dispose of the scalar triplets $\rho$ and $\chi$ in order to generate the charged lepton masses by dimension-five effective operators. According to the transformation properties under the symmetry $S U(3)_{C} \otimes S U(3)_{L} \otimes U(1)_{N}$ of the last two scalar triplets we can form with $\overline{\Psi_{a L}^{c}} \Psi_{b L}$ the following, effective dimensionfive operator:

$$
\begin{aligned}
\mathcal{L}= & \frac{f_{a b}}{\Lambda} \overline{\Psi_{a L}^{c}} \Psi_{b L} \chi^{*} \rho^{*}+\text { H.c. } \\
= & \frac{f_{a b}}{\Lambda}\left\{\overline{\nu_{a L}^{c}}\left[\nu_{b L} \chi^{+} \rho^{-}+l_{b L} \chi^{+} \rho^{0 *}+\left(l^{c}\right)_{b L} \chi^{+} \rho^{--}\right]\right. \\
& +\overline{\left(l_{a L}\right)^{c}}\left[\nu_{b L} \chi^{++} \rho^{-}+l_{b L} \chi^{++} \rho^{0 *}+l_{b L} \chi^{++} \rho^{--}\right] \\
& \left.+\overline{l_{a R}}\left[\nu_{b L} \chi^{0 *} \rho^{-}+l_{b L} \chi^{0 *} \rho^{0 *}+\left(l^{c}\right)_{b L} \chi^{0 *} \rho^{--}\right]\right\} \\
& + \text {H.c. }
\end{aligned}
$$

After the neutral components $\chi_{0}$ and $\rho_{0}$ develop their respective vacuum expectation values (VEVs), $\langle\chi\rangle$ and $\langle\rho\rangle$, the effective operator above generates the following expression for the charged lepton mass matrix:

$$
M_{a b}^{l}=\frac{f_{a b}}{\Lambda}\langle\rho\rangle\langle\chi\rangle
$$

with $a, b=e, \mu, \tau$. Let us discuss the values of the parameters present in the expression above. None of them has already been fixed by the model. We just expect that they can be found in some range of values. For example, $\langle\chi\rangle$ must be in the range $300 \mathrm{GeV}<\langle\chi\rangle<4 \mathrm{TeV}[17,18]$. The constraint on $\langle\rho\rangle$ comes from the mass of the gauge bosons $W^{ \pm}$and $Z^{0}$ i.e., $\langle\rho\rangle^{2}+\langle\eta\rangle^{2}=(246)^{2} \mathrm{GeV}^{2}$. Assuming, as an illustration, the following set of values:

$$
\begin{aligned}
& \langle\eta\rangle \simeq 22 \mathrm{GeV}, \quad\langle\rho\rangle \simeq 245 \mathrm{GeV}, \\
& \langle\chi\rangle \simeq 1 \mathrm{TeV}, \text { and } \Lambda \simeq 5 \mathrm{TeV},
\end{aligned}
$$

the charged lepton mass matrix takes the form

$$
M_{a b}^{l} \simeq 49 f_{a b} \mathrm{GeV} .
$$

If $f_{a b}$ is a diagonal matrix, for obtaining the correct charged lepton masses we need $f_{e e} \sim \times 10^{-5}$ for the electron mass, $f_{\mu \mu} \sim 2 \times 10^{-3}$ for the muon mass, and $f_{\tau \tau} \sim 3.6 \times 10^{-2}$ for the tau mass. We recall that in the case of the standard model we have $10^{-6}, 10^{-3}$, and $10^{-2}$, respectively.

\section{B. Neutrino mass}

In order to generate neutrino masses we will consider only effective operators that conserve the total lepton number and also some other global or/and discrete symmetries. The simplest way to obtain such operator is by adding an scalar singlet $\phi \sim(1,1,0)$, coming from new physics at the $\mathrm{TeV}$ scale, carrying the total lepton number, $L=L_{e}+L_{\mu}+L_{\tau}$, with the following assignment $L(\phi)=-1$, and forming with $\eta$ and $\Psi_{a L}$ the dimension-seven effective operator:

$$
\begin{aligned}
\mathcal{L}= & \frac{f_{a b}^{\prime}}{\Lambda^{3}} \overline{\Psi_{a L}^{c}} \Psi_{b L} \eta^{*} \eta^{*} \phi \phi+\text { H.c. } \\
= & \frac{f_{a b}^{\prime}}{\Lambda^{3}}\left\{\overline{\nu_{a L}^{c}}\left[\nu_{b L} \eta^{0 *} \eta^{0 *}+l_{b L} \eta^{0 *} \eta_{1}^{+}+\left(l^{c}\right)_{b L} \eta^{0 *} \eta_{2}^{-}\right]\right. \\
& +\overline{\left(l_{a L}\right)^{c}}\left[\nu_{b L} \eta_{1}^{+} \eta^{0 *}+l_{b L} \eta_{1}^{+} \eta_{1}^{+}+\left(l^{c}\right)_{b L} \eta_{1}^{+} \eta_{2}^{-}\right] \\
& \left.+\overline{l_{a R}}\left[\nu_{b L} \eta^{0 *} \eta_{2}^{-}+l_{b L} \eta_{2}^{-} \eta_{1}^{+}+\left(l^{c}\right)_{b L} \eta_{2}^{-} \eta_{2}^{-}\right]\right\} \phi \phi \\
& + \text { H.c. }
\end{aligned}
$$

Notice that this operator conserves the total lepton number, since $L\left(\eta_{2}^{+}\right)=-2$ and $L\left(\eta_{1}^{-}\right)=L\left(\eta^{0}\right)=0$ [we recall that $L\left(\chi^{-}\right)=L\left(\chi^{--}\right)=2$ and hence the interactions in Eq. (4) are also $L$ conserving]. The dimension-five operator $\overline{\Psi_{a L}^{c}} \Psi_{b L} \eta^{*} \eta^{*}$ which violates the lepton number explicitly can be forbidden by introducing discrete symmetries as we will show below. For the moment we will avoid it. If we want this quantum number to be broken spontaneously we should begin with $L$-conserving interactions and let a nonzero VEV, in this case $\langle\phi\rangle$, break this symmetry spontaneously. A dangerous Majoron-like Goldstone can be avoided by breaking softly or explicitly the total lepton number in the scalar potential [see Eq. (11) below] or by assuring that the Majoron is almost singlet [19]. Hence, after the scalars involved in Eq. (8) develop their respective VEVs the neutrino masses are given by

$$
M_{a b}^{\nu}=\frac{f_{a b}^{\prime}}{\Lambda^{3}}\langle\eta\rangle^{2}\langle\phi\rangle^{2}
$$

Inserting the values of the VEV given in Eq. (6) the expression above reads 


$$
M_{a b}^{\nu} \simeq 3.9 f_{a b}^{\prime}\left(\frac{\langle\phi\rangle}{1 \mathrm{GeV}}\right)^{2} \times 10^{-9} \mathrm{GeV} .
$$

According to this the VEV involved above, $\langle\phi\rangle$, has to be around $5 \times 10^{-2} \mathrm{GeV}$ if $f_{a b}^{\prime} \approx O(1)$, in order to generate the expected order of magnitude of the neutrino masses, that is, of the $10^{-2} \mathrm{eV}$ order. That value of the VEV for the scalar singlet could imply a fine-tuning since we expect the singlet scalar boson to be very heavy, i.e., $m_{\phi} \approx \Lambda$. However, in order to get such a small VEV in a more natural way we can implement a type II seesaw mechanism with the scalar field $\phi[20]$.

In fact, it is possible to implement this mechanism as we will show in the following. Let us consider, for the sake of simplicity, the discrete symmetry $\eta \rightarrow-\eta, \quad \phi \rightarrow-\phi$ (other fields are even under this symmetry). We also allow terms in the scalar potential that violate explicitly the total lepton number. In this case the most complete scalar potential presenting these terms is

$$
\begin{aligned}
V(\eta, \rho, \chi, \phi)= & \mu_{\eta}^{2} \eta^{\dagger} \eta+\mu_{\rho}^{2} \rho^{\dagger} \rho+\mu_{\chi}^{2} \chi^{\dagger} \chi+\mu_{\phi} \phi^{\dagger} \phi \\
& +\lambda_{1}\left(\eta^{\dagger} \eta\right)^{2}+\lambda_{2}\left(\rho^{\dagger} \rho\right)^{2}+\lambda_{3}\left(\chi^{\dagger} \chi\right)^{2} \\
& +\lambda_{4}\left(\phi^{\dagger} \phi\right)^{2}+\left(\eta^{\dagger} \eta\right)\left[\lambda_{5}\left(\rho^{\dagger} \rho\right)+\lambda_{6}\left(\chi^{\dagger} \chi\right)\right] \\
& +\lambda_{7}\left(\rho^{\dagger} \rho\right)\left(\chi^{\dagger} \chi\right)+\lambda_{8}\left(\rho^{\dagger} \eta\right)\left(\eta^{\dagger} \rho\right)+\lambda_{9}\left(\chi^{\dagger} \eta\right) \\
& \times\left(\eta^{\dagger} \chi\right)+\lambda_{10}\left(\rho^{\dagger} \chi\right)\left(\chi^{\dagger} \rho\right)+\left(\phi^{\dagger} \phi\right) \\
& \times\left[\lambda_{11}\left(\eta^{\dagger} \eta\right)+\lambda_{12}\left(\rho^{\dagger} \rho\right)+\lambda_{13}\left(\chi^{\dagger} \chi\right)\right] \\
& +\left[\lambda_{14} \epsilon \eta \rho \chi \phi+\lambda_{15} \chi^{\dagger} \eta \rho^{\dagger} \eta+\text { H.c. }\right],
\end{aligned}
$$

where the last two terms are those that explicitly violate the lepton number. From this scalar potential we find the following constraint equation over $\langle\phi\rangle$ :

$$
\begin{aligned}
& \langle\phi\rangle\left[\mu_{\phi}^{2}+\lambda_{11}\langle\eta\rangle^{2}+\lambda_{12}\langle\rho\rangle^{2}+\lambda_{13}\langle\chi\rangle^{2}+\lambda_{4}\langle\phi\rangle^{2}\right] \\
& +\lambda_{14}\langle\eta\rangle\langle\rho\rangle\langle\chi\rangle=0 .
\end{aligned}
$$

Supposing that $\mu_{\phi}^{2}<0$ is the dominant parameter in the term within square brackets, we have,

$$
\langle\phi\rangle \simeq-\lambda_{14} \frac{\langle\eta\rangle\langle\rho\rangle\langle\chi\rangle}{\mu_{\phi}^{2}} .
$$

Using the values in Eq. (6) in Eq. (13), and assuming $\left|\mu_{\phi}\right|$ $\approx \Lambda$ we obtain $\langle\phi\rangle \simeq 5 \times 10^{-2} \mathrm{GeV}$, if $\lambda_{14}=0.25$. We recall that it was already shown in the literature that it is possible to have a heavy scalar with a corresponding small VEV $[10,19]$ as in the present case.

From Eq. (10) we find that the neutrino mass matrix is given by the following expression:

$$
M_{a b}^{\nu} \simeq 10^{-2} f_{a b}^{\prime} \mathrm{eV} .
$$

We see from the discussion above that both charged leptons and neutrinos gain mass through effective operators, as can be seen from Eqs. (5) and (10) or (7) and (14). However, the scale of neutrino masses relative to the charged lepton masses arises as a consequence of the dimension of the effective operator and of the VEV of the scalar involved.

\section{REALISTIC SCENARIOS}

If we want to obtain a definite texture on the mass matrices in Eqs. (5) and (9) we have to enlarge the number of scalar multiplets, for instance one triplet of the type $\rho$ and $\chi$ and a singlet $\phi$ for each generation. We denote them as $\rho_{1,2,3}, \chi_{1,2,3}, \phi_{1,2,3}$. In this case we have

$$
\mathcal{L}=\frac{f_{a b}^{i j}}{\Lambda} \overline{\Psi_{a L}^{c}} \Psi_{b L} \chi_{i}^{*} \rho_{j}^{*}+\frac{f_{a b}^{\prime i j}}{\Lambda^{3}} \overline{\Psi_{a L}^{c}} \Psi_{b L} \eta^{*} \eta^{*} \phi_{i} \phi_{j}+\text { H.c. }
$$

and we will impose discrete symmetries in order to obtain appropriate mass matrices. For instance, consider the symmetry $Z_{9} \otimes Z_{2}$ with fields transforming under the $Z_{9}$ factor as

$$
\begin{gathered}
\Psi_{e} \rightarrow \omega_{1} \Psi_{e}, \quad \Psi_{\mu} \rightarrow \omega_{3} \Psi_{\mu}, \quad \Psi_{\tau} \rightarrow \omega_{2} \Psi_{\tau}, \\
\rho_{1} \rightarrow \omega_{3} \rho_{1}, \quad \chi_{1} \rightarrow \omega_{1}^{-1} \rho_{1}, \\
\rho_{2} \rightarrow \omega_{3}^{-1} \rho_{2}, \quad \chi_{2} \rightarrow \omega_{0} \chi_{2}, \quad \rho_{3} \rightarrow \omega_{4} \rho_{3}, \\
\chi_{3} \rightarrow \omega_{4}^{-1} \chi_{3}, \quad \eta \rightarrow \omega_{0} \eta, \\
\phi_{1} \rightarrow \omega_{1}^{-1} \phi_{1}, \quad \phi_{2} \rightarrow \omega_{3}^{-1} \phi_{2}, \quad \phi_{3} \rightarrow \omega_{2}^{-1} \phi_{3},
\end{gathered}
$$

with $\omega_{k}=e^{2 \pi i k / 9}, k=0, \ldots, 4$ and under the $Z_{2}$ factor the fields $\Psi_{\mu}, \rho_{1}, \chi_{1}, \phi_{2,3}$ are odd, while the other ones are even. (This implies appropriate transformation in the quark sector if the scalar multiplets also couple to quarks.) Notice that the $Z_{9}$ symmetry forbids the interactions $\epsilon \overline{\Psi_{a L}^{c}} \Psi_{b L} \eta$ and $\overline{\Psi_{a L}^{c}} \Psi_{b L} \eta^{*} \eta^{*}$.

From the interactions in Eq. (15) and the discrete symmetries in Eq. (16) we obtain mass matrices which mix the second and third generations in the charged lepton sector, and the first and the second generations in the neutrino sector, i.e., they are diagonalized by the following orthogonal transformations:

$$
U_{L}^{l} \cong\left(\begin{array}{ccc}
1 & 0 & 0 \\
0 & c_{0} & s_{0} \\
0 & -s_{0} & c_{0}
\end{array}\right), \quad U_{L}^{v} \cong\left(\begin{array}{ccc}
c_{0}^{\prime} & s_{0}^{\prime} & 0 \\
-s_{0}^{\prime} & c_{0}^{\prime} & 0 \\
0 & 0 & 1
\end{array}\right)
$$

for the charged lepton and neutrinos, respectively. The MakiNakagawa-Sakata (MNS) mixing matrix is of the form [21]

$$
U=U_{L}^{l \dagger} U_{L}^{\nu}=\left(\begin{array}{ccc}
c_{0}^{\prime} & s_{0}^{\prime} & 0 \\
-c_{0} s_{0}^{\prime} & c_{0} c_{0}^{\prime} & s_{0} \\
s_{0} s_{0}^{\prime} & -s_{0} c_{0}^{\prime} & c_{0}
\end{array}\right),
$$

and we have omitted Dirac or Majorana $C P$ violating phases. Notice that $U_{e 3}$ is zero at the tree level, and it must 
arise from radiative corrections. After taking into account these corrections we expect that $U \rightarrow V$ :

$$
\begin{aligned}
V & \approx\left(\begin{array}{ccc}
c_{\odot} & s_{\odot} & V_{e 3} \\
-c_{\mathrm{atm}} s_{\odot} & c_{\mathrm{atm}} c_{\odot} & -s_{\mathrm{atm}} \\
-s_{\mathrm{atm}} s_{\odot} & s_{\mathrm{atm}} c_{\odot} & c_{\mathrm{atm}}
\end{array}\right) \\
& \simeq\left(\begin{array}{ccc}
c_{\odot} & s_{\odot} & V_{e 3} \\
-\frac{1}{\sqrt{2}} s_{\odot} & \frac{1}{\sqrt{2}} c_{\odot} & -\frac{1}{\sqrt{2}} \\
-\frac{1}{\sqrt{2}} s_{\odot} & \frac{1}{\sqrt{2}} c_{\odot} & \frac{1}{\sqrt{2}}
\end{array}\right) .
\end{aligned}
$$

In this context, the small value of $\left|V_{e 3}\right|(<0.16)$ [22] is natural in the present model because it arises from radiative corrections involving a term in the scalar potential which softly breaks those discrete symmetries. For instance, in a generalization of the expression in Eq. (11) a term like $\chi_{1}^{\dagger} \chi_{2}$ softly breaks the $Z_{9}$ symmetry. Hence, radiative corrections should induce a small value for this entry in the MNS matrix and (small) corrections to the angles in Eqs. (17) and (18). In this case $(c, s)_{0} \rightarrow(c, s)_{\mathrm{atm}},\left(c^{\prime}, s^{\prime}\right)_{0} \rightarrow(c, s,)_{\odot}, \quad U_{e 3}(=0)$ $\rightarrow V_{e 3}(\leqslant 0.16)$. Since in general we expect that radiative correction does not amplify the mixing angles in Eqs. (17) and (18) then $(c, s)_{0} \simeq(c, s)_{\text {atm }}$ and $\left(c^{\prime}, s^{\prime}\right)_{0} \simeq(c, s)_{\odot}$. Notice that the interactions that contribute to a nonzero value for $V_{e 3}$ may induce a complex value for this entry inducing in this way a $C P$ violating phase [23].

Another possible scenario, where there is bimaximal mixing among the neutrinos but not in the MNS matrix, appears as a result of a new symmetry $L^{\prime}$ (in the context of the standard model $L^{\prime}$ can be identified with $L_{e}-L_{\mu}-L_{\tau}$ $[12,24,25]$ but here it must be an independent global symmetry). In this case we need only two sorts of $\rho$ and $\chi$ scalar triplets and two singlets. We assign $L^{\prime}\left(\Psi_{e}\right)=-L^{\prime}\left(\Psi_{\mu}\right)=$ $-L^{\prime}\left(\Psi_{\tau}\right)=1, \quad L^{\prime}\left(\rho_{1}\right)=L^{\prime}\left(\chi_{1}\right)=-L^{\prime}\left(\rho_{2}\right)=-L^{\prime}\left(\chi_{2}\right)=1$, and $L^{\prime}(\eta)=L^{\prime}(\phi)=0$. With this symmetry we obtain from Eq. (15) a general mass matrix in the charged lepton sector, and the following neutrino mass matrix at the tree level:

$$
M^{\nu}=\left(\begin{array}{ccc}
0 & f_{e \mu}^{\prime 12} & f_{e \tau}^{\prime 12} \\
f_{e \mu}^{\prime 12} & 0 & 0 \\
f_{e \tau}^{\prime 12} & 0 & 0
\end{array}\right) \frac{\langle\eta\rangle^{2}\left\langle\phi_{1}\right\rangle\left\langle\phi_{2}\right\rangle}{\Lambda^{3}}
$$

with $f_{e \mu}^{\prime 12} \sim f_{e \tau}^{\prime 12}$. In this case the neutrino mass matrix has the inverse hierarchy $(m,-m, 0)$ where $m \propto\left[\left(f_{e \mu}^{\prime 12}\right)^{2}\right.$ $\left.+\left(f_{e \tau}^{\prime 12}\right)^{2}\right]^{1 / 2}$. The mass splitting has to be generated by radiative corrections, as we will show below, and we have $\left|m_{3}\right| \ll\left|m_{1}\right| \simeq\left|m_{2}\right|$. Assuming that $f_{e \mu}^{\prime 12}=f_{e \tau}^{\prime 12}$ there is a bimaximal neutrino mixing pattern [24]:

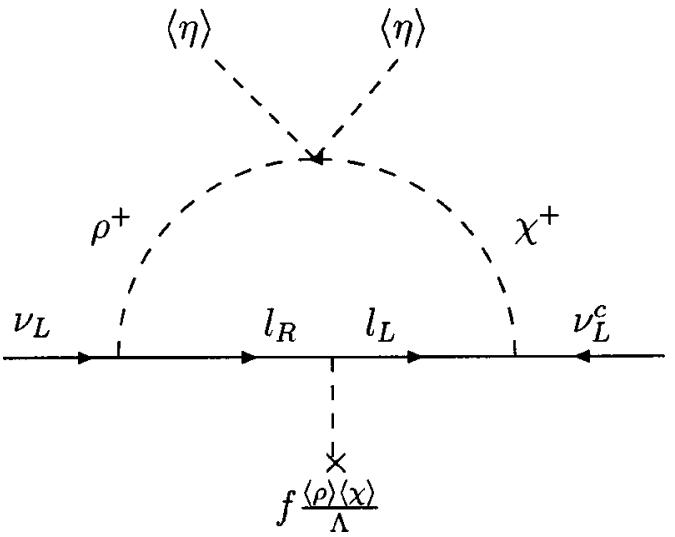

FIG. 1. One-loop contribution to the diagonal entries for the neutrino mass matrix in the second almost bimaximal scenario.

$$
U_{L}^{\nu}=\left(\begin{array}{ccc}
\frac{1}{\sqrt{2}} & -\frac{1}{\sqrt{2}} & 0 \\
\frac{1}{2} & \frac{1}{2} & -\frac{1}{\sqrt{2}} \\
\frac{1}{2} & \frac{1}{2} & \frac{1}{\sqrt{2}}
\end{array}\right) \text {. }
$$

Since the bimaximal mixing is not favored by the actual neutrino solar data, i.e., $\tan \theta_{\odot}<1$ [5], we have to explain the deviation from this bimaximal scenario. This can be done since from Eq. (15) and the $L^{\prime}$ symmetry the mass matrix in the charged lepton sector, as we said before, is now a general one. Hence the left-handed mixing matrix $U_{L}^{l}$ can be written in terms of three angles $(c, s)_{12},(c, s)_{23},(c, s)_{13}$. In this case assuming that $s_{12} \gg s_{23} \gg s_{13}$ the mixing matrix $U_{L}^{l}$ is a hierarchical one similar to the mixing matrix in the quark sector and the charged lepton mass matrix is almost diagonal. Since the MNS matrix is defined as $V=U_{L}^{l \dagger} U_{L}^{\nu}$ the analysis of Ref. [26] follows.

In this almost bimaximal scenario we should address the mass splitting between $\nu_{1}$ and $\nu_{2}$. To get such a mass splitting we have to consider terms in the scalar potential that break $L$ explicitly, as is the case of the last $\lambda_{15}$ term in the scalar potential in Eq. (11). However, with the symmetry $Z_{9}$ introduced above, this term involving $\rho_{i}, \chi_{i}$, and $\eta$ is forbidden. Hence, it should be necessary that $\eta$ transforms nontrivially under $Z_{9}$ (or a higher discrete symmetry) or, only as an illustration, we can add a fourth pair of triplets $\rho_{4}$ and $\chi_{4}$ which transform like $\eta$ (and for this reason they do not couple directly with leptons) and with $L^{\prime}\left(\chi_{4}\right)=L^{\prime}\left(\rho_{4}\right)=0$. Thus we have the term $\lambda_{15}\left(\chi_{4}^{\dagger} \eta\right)\left(\eta^{\dagger} \rho_{4}\right)$. The scalar potential produces a general mixing among all the scalar of the same charge and we have interactions like $\chi^{+} \rho^{-}\langle\eta\rangle^{2}$, where $\chi^{+}, \rho^{-}$denote symmetry eigenstates. That term, together with the interaction in Eq. (15), will generate corrections, through the one-loop diagrams, for example to the diagonal entries in the mass matrix in Eq. (20), providing the mass splitting between $\nu_{1}$ and $\nu_{2}$. The loop diagram which will generate the diagonal entries in Eq. (20) is depicted in Fig. 1. 
It gives, up to logarithmic corrections, the following expression for such entries:

$$
M_{a a}^{\nu} \simeq \frac{\lambda_{15} f_{a a}^{3}\langle\eta\rangle^{2}\langle\rho\rangle^{2}\langle\chi\rangle^{2}}{m_{\chi}^{2} \Lambda^{3}}
$$

All the parameters above except $m_{\chi}$ and $\lambda_{15}$ were already previously fixed in this work. Assuming now that $m_{\chi}=\langle\chi\rangle$, and inserting Eq. (6) in Eq. (22), we have

$$
M_{a a}^{\nu} \simeq 1.1 \times 10^{-8} \lambda_{15} f_{a a}^{3} \mathrm{GeV} .
$$

The large mixing angle Mikheyev-Smirnov-Wolfenstein (MSW) solution to the solar neutrino problem requires a neutrino mass scale of the order of $2.8 \times 10^{-3} \mathrm{eV}$ [27]. The only free parameter in Eq. (23) is $\lambda_{15}$, while the diagonal $f$ parameters are already fixed by the charged lepton masses and their values are given in Sec. II A (although in this case each entry of the matrix $f_{a b}$ has several contributions that we have not written explicitly). We obtain this mass scale for the neutrinos by considering $\lambda_{15} \sim 2.5 \times 10^{-4}$. Hence to generate the $\nu_{1}-\nu_{2}$ mass splitting we must fine-tune $\lambda_{15}$.

In the next section we analyze what main ingredients an underlying theory should have to realize, in an economical way, the effective operators and generate the two mixing scenarios considered above.

\section{A POSSIBLE UNDERLYING THEORY}

The minimal scenario we can imagine is the one where the effective dimension-five operator in Eq. (4) is realized at the tree level, while the effective dimension-seven operator in Eq. (8) is realized through the one-loop level. For this we only need to add to the minimal 3-3-1 model (three quark generations and three scalar triplets $\eta, \rho$, and $\chi$ ) at least two sextets $S^{p}, p=1,2$, which do not necessarily gain a nonzero $\mathrm{VEV}$, two heavy lepton singlets, $E_{1,2(L, R)} \sim(\mathbf{1}, \mathbf{1},-1)$ $[15,16]$, and three scalar singlets $\phi^{r}, r=1,2,3$, like the $\phi$ introduced in Sec. II B. We will assume that these singlet leptons have the following assignments of the total lepton number: $L\left(E_{1}\right)=1$ and $L\left(E_{2}\right)=0$. We recall that it was shown in Ref. [28] that a tree level realization of a symmetric bilinear $\overline{\Psi_{L}^{c}} \Psi_{L}$ is implemented by introducing a scalar sextet.

With the representation content discussed above we have the leptonic interactions

$$
\begin{aligned}
\mathcal{L}= & G_{a b}^{p} \overline{\left(\Psi^{c}\right)_{a i L}} \Psi_{b j L} S_{i j}^{p}+G_{1 a} \overline{\Psi_{a L}} E_{1 R} \rho+G_{2 a} \chi^{T} \overline{N_{1 L}} \Psi_{a L}^{c} \\
& +g_{r}^{\prime} \overline{E_{1 L}} E_{2 R} \phi^{r}+g_{r} \overline{E_{2 L}} E_{1 R} \phi^{r *}+M_{1} \overline{E_{1 L}} E_{1 R} \\
& +M_{2} \overline{E_{2 L}} E_{2 R}+\text { H.c. }
\end{aligned}
$$

with $M_{1}, M_{2} \simeq \Lambda$ and we have omitted $S U$ (3) indices and summation over the repeated indices. By imposing an appropriate discrete symmetry we can get the result that one of the scalar sextets couples only to the first leptonic generation and the other one to the second and third generations. Hence,
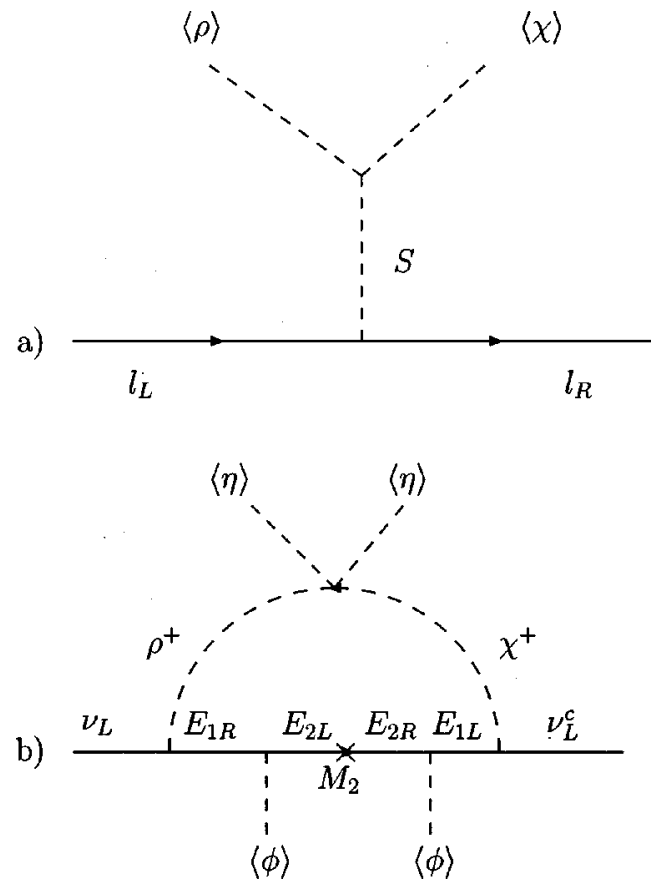

FIG. 2. Tree level and one-loop diagrams contributing to the effective operators defined in Eqs. (4) and (8), respectively.

with the interactions above we can realize the effective dimension-five operator in Eq. (15) as is shown in Fig. 2(a), where the trilinear vertex arises from a term like $g \chi^{T} S^{\dagger} \rho$ where $g$ is a constant with dimension of mass. So, we obtain a mass matrix for the charged lepton which is diagonalized by an orthogonal matrix, like $U_{L}^{l}$ in Eq. (17), and from Eq. (4) we see that $1 / \Lambda=g / M_{S}^{2}$. For the realization of the effective dimension-seven operator the last term of the scalar potential in Eq. (11) is also important. This realization is depicted in Fig. 2(b).

\section{CONCLUSIONS}

In this work we developed a simple mechanism based on effective operators in the context of a 3-3-1 model which generates masses for the neutrinos and for the charged leptons as well. By using the same bilinear the charged lepton masses are generated in this mechanism by an effective dimension-five operator, while the neutrino masses require an effective dimension-seven operator in conjunction with a type II seesaw mechanism applied on a scalar singlet. Then, we use the effective operator mechanism to generate several mixing matrices in the lepton sector which are consistent with the solar, atmospheric, and reactor neutrino data. For this we have considered the discrete symmetry $Z_{9} \otimes Z_{2}$ or a global one, $L^{\prime}$, in the effective operators given in Eq. (15). In this case, without resort to large fine-tuning we obtain neutrino masses compatible with some solutions to the solar and atmospheric neutrino anomalies. We would like to stress that in this 3-3-1 model the bilinear $\overline{\Psi^{c}} \Psi$ gives mass to both lepton sectors. This issue is characteristic of the 3-3-1 models: in the standard model the bilinear $\overline{L^{c}} L$ can only contribute to the neutrino masses. 
Although we have not considered the quark masses we would like to call attention to an interesting mechanism, in the context of a 3-3-1 model, for generating the top and bottom masses at the tree level, while the masses of the other quarks and charged leptons arise at the one-loop level, as proposed in Ref. [29].

\section{ACKNOWLEDGMENTS}

This work was supported by Fundação de Amparo à Pesquisa do Estado de São Paulo (FAPESP), Conselho Nacional de Ciência e Tecnologia (CNPq), and Programa de Apoio a Núcleos de Excelência (PRONEX).
[1] Y. Fukuda et al., Phys. Rev. Lett. 81, 1562 (1998); 81, 1158 (1998); Phys. Lett. B 436, 33 (1998); K. S. Hirata et al., ibid. 280, 146 (1992); R. Becker-Szendy et al., Phys. Rev. D 46, 3720 (1992); W. W. M. Allison et al., Phys. Lett. B 391, 491 (1997); Y. Fukuda et al., ibid. 335, 237 (1994).

[2] Homestake Collaboration, B. T. Cleveland et al., Nucl. Phys. B (Proc. Suppl.) 38, 47 (1995); K. S. Hirata et al., Phys. Rev. D 44, 2241 (1991); GALLEX Collaboration, W. Hampel et al., Phys. Lett. B 477, 127 (1999); SAGE Collaboration, J. N. Abdurashitov et al., Phys. Rev. Lett. 77, 4708 (1996).

[3] SNO Collaboration, Q. R. Ahmed et al., Phys. Rev. Lett. 87, 071301 (2001).

[4] For an extensive review and references see S. Bilenky et al., in Proceedings of the Workshop on Neutrinos NOW98, hep-ph/9906251; see also G. Altarelli and F. Feruglio, hep-ph/0106085; R. N. Mohapatra, hep-ph/9910365; F. Vissani, hep-ph/9708483. For possible matrix textures see S. M. Barr and I. Dorsner, Nucl. Phys. B585, 79 (2000); B. R. Desai, U. Sarkar, and A. R. Vaucher, hep-ph/0007346; R. Barbieri, L. J. Hall, and A. Strumia, Phys. Lett. B 445, 407 (1999).

[5] Super-Kamiokande Collaboration, M. B. Smy, hep-ex/0208004.

[6] R. N. Mohapatra and G. Senjanovic, Phys. Rev. D 23, 165 (1981); C. Wetterich, Nucl. Phys. B187, 343 (1981); E. Ma and U. Sarkar, Phys. Rev. Lett. 80, 5716 (1998); G. B. Gelmini and M. Roncadelli, Phys. Lett. 99B, 411 (1981); R. N. Mohapatra and G. Senjanović, Phys. Rev. D 23, 165 (1981); C. Wetterich, Nucl. Phys. B187, 343 (1981); E. Ma and U. Sarkar, Phys. Rev. Lett. 80, 5716 (1998).

[7] K. S. Babu, Phys. Lett. B 203, 132 (1988); A. Zee, Phys. Lett. 93B, 389 (1980).

[8] S. Weinberg, Phys. Rev. Lett. 43, 1566 (1979).

[9] F. Wilczek and A. Zee, Phys. Rev. Lett. 43, 1571 (1979).

[10] E. Ma, Phys. Rev. Lett. 81, 1171 (1998).

[11] K. S. Babu and C. N. Leung, Nucl. Phys. B619, 667 (2001).

[12] See, for instance, R. N. Mohapatra and S. Nussinov, Phys. Rev. D 60, 013002 (1999); R. N. Mohapatra, A. Perez-Lorenzana, and C. A. de S. Pires, Phys. Lett. B 474, 355 (2000); A. Joshipura and S. Rindani, Eur. Phys. J. C 14, 85 (2000); Phys. Lett. B 464, 239 (1999).

[13] F. Pisano and V. Pleitez, Phys. Rev. D 46, 410 (1992); P. H. Frampton, Phys. Rev. Lett. 69, 2889 (1992).

[14] R. Foot, O. F. Hernández, F. Pisano, and V. Pleitez, Phys. Rev. D 47, 4158 (1993).
[15] T. V. Duong and E. Ma, Phys. Lett. B 316, 307 (1993).

[16] J. C. Montero, C. A. de S. Pires, and V. Pleitez, Phys. Rev. D 65, 093017 (2002).

[17] D. Ng, Phys. Rev. D 49, 4805 (1994).

[18] P. H. Frampton, hep-ph/0208044.

[19] J. C. Montero, C. A. de S. Pires, and V. Pleitez, Phys. Rev. D 60, 098701 (1999).

[20] A. Perez-Lorenzana and C. A. de S. Pires, Phys. Lett. B 522, 297 (2001); J. C. Montero, C. A. de S. Pires, and V. Pleitez, ibid. 502, 167 (2001); M. B. Tully and G. C. Joshi, Phys. Rev. D 64, 011301(R) (2001).

[21] S. M. Barr, hep-ph/0206085.

[22] M. Apollonio et al., Phys. Lett. B 466, 415 (1999); F. Boehm et al., Phys. Rev. D 62, 072002 (2000); 64, 112001 (2001).

[23] T. Miura, T. Shindou, and E. Takasugi, Phys. Rev. D 66, 093002 (2002).

[24] H. Fritzsch and Z. Xing, Phys. Lett. B 372, 265 (1996); 440, 313 (1998); F. Vissani, hep-ph/9708483; V. Barger, S. Pakvasa, T. Weiler, and K. Whisnant, Phys. Lett. B 437, 107 (1998); A. Baltz, A. S. Goldhaber, and M. Goldhaber, Phys. Rev. Lett. 81, 5730 (1998); M. Jezabek and Y. Sumino, Phys. Lett. B 440, 327 (1998); G. Altarelli and F. Feruglio, ibid. 439, 112 (1998); S. Davidson and S. F. King, ibid. 445, 191 (1998); C. S. Kim and J. D. Kim, Phys. Rev. D 61, 057302 (2000); C. H. Albright and S. Barr, Phys. Lett. B 461, 218 (1999); H. Georgi and S. L. Glashow, Phys. Rev. D 61, 097301 (2000); H. B. Benaoum and S. Nasri, ibid. 60, 113003 (1999); M. Jezabek and Y. Sumino, Phys. Lett. B 457, 139 (1999); R. Barbieri, L. Hall, A. Strumia, and N. Weiner, J. High Energy Phys. 12, 017 (1998); R. Barbieri, L. J. Hall, and A. Strumia, Phys. Lett. B 445, 407 (1999); Y. Grossman, Y. Nir, and Y. Shadmi, J. High Energy Phys. 10, 007 (1998); R. Barbieri, hep-ph/9901241; G. Altarelli and F. Feruglio, Phys. Rep. 320, 295 (1999); E. Kh. Akhmedov, Phys. Lett. B 467, 95 (1999); E. Kh. Akhmedov, G. C. Branco, and M. N. Rebelo, ibid. 478, 215 (2000).

[25] For bimaximal mixing in 3-3-1 models, see T. Kitabayashi and M. Yasue, Phys. Rev. D 63, 095006 (2001); Y. Okamoto and M. Yasue, Phys. Lett. B 466, 267 (1999); T. Kitabayashi and M. Yasue, hep-ph/0006040; hep-ph/0011153; Nucl. Phys. B609, 61 (2001).

[26] C. Giunti and M. Tanimoto, Phys. Rev. D 66, 053013 (2002).

[27] A. Gago et al., Phys. Rev. D 65, 073012 (2002).

[28] J. C. Montero, C. A. de S. Pires, and V. Pleitez, Phys. Rev. D 65, 095001 (2002).

[29] M. D. Tonasse, Nucl. Phys. B623, 316 (2002). 